\title{
Osteopathy and pubalgy
}

\begin{abstract}
Pubalgy is the result of excessive and repetitive overuse of the pubic symphysis may implicate a prolonged interruption of physical activity. This can also be known as pubic osteitis, since pain occurs in surrounding structures of the pubic symphysis. The pubic bone is the place where some muscle attaches and their tendons become inflamed due to repetitive stress over that region. Many athletes, football players, runners and others, feel an uncomfortable pain in the pubic symphysis, caused by overload, excessive use, direct trauma or any other blow that may produce inflammation and pain.
\end{abstract}

Volume I Issue 3 - 2017

\section{Marcio Domingues}

Universidade Lusófona, Portugal

Correspondence: Marcio Domingues, Universidade Lusófona, Portugal, Tel (+35I)2I75I5500, Fax (+35I)217577006,

Email marcio.domingues@live.com.pt

Received: May 30, 2017 | Published: September 07, 2017

\section{Introduction}

Athletes are predisposed to this kind of injuries due to their repetitive natures and high volume of games with short intervals for rest and recovery. The lack or inadequate stretching of the adductor muscles combined with an overload of abdominal exercise that athletes often do, can cause a muscular imbalance in the pubic symphysis and therefore, Pubalgy. On the other side, there are factors that are intrinsic to each person, for example, congenital or acquired abnormalities from the abdominal wall, especially in its lower quadrants, inguinal channel abnormalities, differences in leg length; they all add some pelvic instability.

There are three kinds of pubalgies. The first, athlete's abdominal parietal Pubalgy mainly in athletes with less than 30 years and football players but it can happen in other sports, namely, fencing, rugby, horse ride, athletics and martial arts. It has a very specific etiology and originates from an imbalance between the abdominals and the adductors, as mentioned previously in the text, with the latter tending to be stronger. This imbalance creates a lack of stability in the pubic region and favors movements of translation, which obviously originates pain. A typical gesture that overloads these structures is the football kick. A second kind is the osteoarthropaty it originates from a bony inflammation (periosteum) due to a compensated short leg, growth or birth anomaly. Pain usually centers around the pubic bone. Finally, adductor tendinitis: situated alongside the insertion, in the tendon or in the junction area of the tendon and muscle.

In fact, pain grows in intensity when the disease starts to worsen. Its beginning is insidious, progressively growing until the moment where movements become limited. With time, pain that once were locates only in the hips, migrate to other parts such as the abdomen and groin. It can easily be misjudged with other problems like herniation in the groin, ruptures, urinary infection and adductor tendinitis. Symptoms are very much the same as a muscle strain, occurring during running, abdominal exercise and squats. Pain can also occur in the inferior abdominal region, radiating to the medial side of the thigh. Pain can also be felt in the insertion point of the rectus abdominal. Lateral movements, heading movements, hip and trunk flexion and might also be painful. This pain worsens with exercise, stress or with some postures; it can also be felt climbing stairs or pushing the hip forward. Pain can irradiate to the perineum and testicles and can cause lumbago when associated with Sacro-iliac dysfunction.
The diagnosis can take time, due to the great number of disturbances that affect the hip region, confusing the symptoms and delaying the diagnosis. From a big point of view, the diagnosis is clinic, being further confirmed by X-Ray of the pelvic girdle, to observe the presence of instability of the pubic symphysis in more than $2 \mathrm{~mm}$. The $\mathrm{X}$-Ray may also reveal the presence of bone thickening, a signal flag that reveals the presence of inflammation. Pubalgy is characterized by a prolonged abstinence from three to nine months, being rest a decisive standpoint. ${ }^{1,2}$ Obviously, as soon as the treatment is initiated, better the result. As it is well known, conservative treatment is based on rest, ice and ultra sound, non-steroid anti-inflammatory drugs and peri-local steroids. After the acute phase, one can use compress of hot water over the region that can promote vasodilatation, beneficial to the decrease of pain and to promote adjacent muscle relaxation.

Once the pain is offset, stretching exercises can be introduced in the inner thigh muscles; exercises that strengthen abdominal and lumbar region, as well as find the correct equilibrium between flexors and femoral extensors of the hip; stabilization of the vertebral column. In some cases, we can have a blocked iliac and the re-establishment of its mobility is one important concern and it can be released using manipulative techniques (thrust HVLA) and mobilization techniques. Flexibility gains in all muscles involved directly and indirectly with the pubis: hamstrings, femoral muscle and adductor, external rotators of the hip and quadratus lumborum is important for the overall gains. As in football the imbalances created by the abductors and internal rotators of the hip. Osteopath will examine your posture and area of pain, a variety of combined gentle and safe techniques, including soft tissue massage, spinal manipulation, myo-fascial dry needling, mobilization as well as postural and ergonomic support. Myofascial techniques with joint mobilizations in hip and the Sacro-iliac joints is a mandatory approach. Osteopathic treatment will also work on reducing muscle spasm and easing the strain of the pubic joint ligaments. It is also useful to use hot approach over the muscle region, to promote vasodilation and diminish pain.

\section{Acknowledgements}

None.

\section{Conflict of interest}

Author declares there is no conflict of interest in publishing the article. 


\section{References}

1. Batt ME, Shane JM, Dillingham M. Osteitis pubis in collegiate football players. Med Sci Sports Exerc. 1995;27(5):629-633.
2. Sing R, Cordes R, Silberski D. Osteitis pubis in the active patient. Physician and Sportsmedicine. 1995;23(12):66-73. 